\title{
Aprovechamiento de flora apícola en dos colmenares \\ localizados en los cantones La Concordia y Mocache, Ecuador
}

\section{Use of bee flora in two apiaries located in La Concordia and Mocache cantons, Ecuador}

1 Miguel Ángel Guallpa Calva https://orcid.org/0000-0001-5392-036X Escuela Superior Politécnica de Chimborazo, Chimborazo-Riobamba miguel.guallpa@espoch.edu.ec

2 Jorge Marcelo Caranqui Aldaz https://orcid.org/0000-0001-7555-1294 Escuela Superior Politécnica de Chimborazo, Chimborazo-Riobamba jcaranqui@espoch.edu.ec

3 Armando Esteban Espinoza Espinoza iD https://orcid.org/0000-0002-8566-6594 Escuela Superior Politécnica de Chimborazo, Chimborazo-Riobamba armando.espinoza@espoch.edu.ec

4 Víctor Manuel Espinoza iD https://orcid.org/0000-0001-6058-2274 Escuela Superior Politécnica de Chimborazo, Chimborazo-Riobamba victor.espinoza@espoch.edu.ec

Artículo de Investigación Científica y Tecnológica Enviado: 08/11/2021

Revisado: $23 / 11 / 2021$

Aceptado: $13 / 12 / 2021$

Publicado:05/01/2022

DOI: https://doi.org/10.33262/ap.v4i1.129

Guallpa Calva, M. Ángel, Caranqui Aldaz, J. M., Espinoza Espinoza, A. E., \& Espinoza, V. M. (2022). Aprovechamiento de flora apícola en dos colmenares localizados en los cantones La Concordia y Mocache, Ecuador. AlfaPublicaciones, 4(1), 28-45. https://doi.org/10.33262/ap.v4i1.129 


\section{Palabras}

claves:

Recursos

apícolas, fenología, apiario, sistemas de uso de la tierra

\section{Keywords:}

Beekeeping resources, phenology, apiary, land use systems

\section{Resumen}

Introducción. Antes de planificar actividades de manejo que permitan incrementar la producción apícola, es necesario datos de los elementos florísticos que integran los sistemas de uso de la tierra adyacentes a los colmenares, en esta ocasión para dos unidades productivas de las zonas 4 y 5 de Ecuador. Objetivos. Reconocer especies vegetales con potencial apícola, y estimar su abundancia más la durabilidad de la floración. Metodología. Con el empleo de los métodos: documental, y de campo, para el reconocimiento taxonómico de plantas melíferas, su cantidad, más el monitoreo del florecimiento de cada especie apícola. El análisis estadístico con la aplicación de la prueba de Mann Whitney a las variables abundancia y durabilidad de la floración. Resultados. Las principales familias botánicas con especies nectaríferas y poliníferas en los sitios de estudio son; Fabaceae, Anacardiaceae, Asteraceae y Capparaceae. Para el Colmenar C1 son 14 especies, pertenecientes a 12 familias, por su parte para el colmenar $\mathrm{C} 2$ con 22 especies, pertenecientes a 14 familias botánicas. La oferta del recurso floral tiene un comportamiento similar de abundancia de plantas entre los colmenares $\mathrm{C} 1$ y $\mathrm{C} 2$ a pesar de poseer mayor cantidad de especies con potencial apícola el colmenar C2, lo cual obedece al manejo que se aplica a las áreas que integran los diferentes sistemas de uso del suelo de cada predio. Conclusión. Finalmente, la duración de las flores de las especies que ofertan alimento a las abejas de los colmenares C1 y C2 oscila de 1 a 12 meses durante el año 2020 con predominancia para el colmenar C1 de Cordia alliodora, Erythrina edulis, Senna alata, Inga edulis, Persea americana, Hibiscus rosa-sinensis, Piper aduncum, y Cestrum racemosum que florecen entre 10 a 12 meses. En cambio, en el colmenar C2; Tridax procumbens, Pelargonium sp, y Hydrangea $s p$ florecen durante todo el año.

\section{Abstract}

Introduction. Before planning management activities that allow increasing bee production, data is needed on the floristic elements that make up the land use systems adjacent to the apiaries, this time for two productive units in zones 4 and 5 of Ecuador. Objectives. Recognize plant species with beekeeping potential and estimate their abundance plus the durability of flowering. Methodology. With the use of methods: documentary, and field, for the taxonomic 
recognition of honey plants, their quantity, plus the monitoring of the flowering of each bee species. Results. The main botanical families with nectariferous and polyniferous species in the study sites are Fabaceae, Anacardiaceae, Asteraceae, and Capparaceae. For apiary $\mathrm{C} 1$ there are 14 species, belonging to 12 families, while for apiary $\mathrm{C} 2$ there are 22 species, belonging to 14 botanical families. The supply of the floral resource has a similar behavior of abundance of plants between apiaries $\mathrm{C} 1$ and $\mathrm{C} 2$ despite having a greater number of species with beekeeping potential in apiary $\mathrm{C} 2$, which is due to the management that is applied to the areas that make up the different systems of land use of each property. Conclusion. Finally, the duration of the flowers of the species that offer food to the bees of apiaries $\mathrm{C} 1$ and $\mathrm{C} 2$ ranges from 1 to 12 months during the year 2020 with a predominance for the apiary $\mathrm{C} 1$ of Cordia alliodora, Erythrina edulis, Senna alata, Inga edulis, Persea americana, Hibiscus rosa-sinensis, Piper aduncum, and Cestrum racemosum that flower between 10 to 12 months. On the other hand, in apiary C2; Tridax procumbens, Pelargonium sp, and Hydrangea sp bloom throughout the year.

\section{Introducción}

La producción apícola en Ecuador es fundamental, debido a que se cuenta con climas y flora, en diversas regiones del país, particularmente en los cantones La Concordia y Mocache, son lugares favorables para este sistema productivo y tiene una gran ventaja competitiva al poseer únicamente dos estaciones climatológicas de invierno y verano. Conocer los elementos florísticos que integran los sistemas de uso de la tierra adyacentes a los colmenares, es la primera fase para diseñar actividades de manejo que permitan desarrollar la producción apícola. Actualmente, hay insuficiente información disponible de apiarios, lo cual incide en la planificación de actividades de manejo de los productos apícolas (Agrocalidad, 2015).

Frente a lo expuesto, el Ministerio de Agricultura Acuacultura y Pesca ([MAGAP], 2015) a nivel nacional ha establecido estrategias desde el año 2015 para promover la Apicultura, debido a la alta demanda de estos productos que ayudan a la Seguridad Alimentaria y también por el alarmante escenario del declive de las abejas a nivel mundial, por lo que se ha tomado iniciativas viables para esta actividad llegando a denominar el año 2015 como el Año de la Apicultura. 
Para ello, el proyecto Impulso a la producción apícola a partir de la constitución de sistemas agroecológicos, con el grupo de investigación llamado "Conservación y Producción sustentable de los Recursos Naturales y Entomológicos (COPROSURENE)" de la Escuela Superior Politécnica de Chimborazo (ESPOCH), ejecuta investigaciones, que incluye el desarrollo e innovación, en aras de incrementar su productividad y competitividad con base en la caracterización de la flora con potencial melífero (Guallpa et al., 2020; Flórez y Ward, 2013).

Por lo expuesto, la disponibilidad de los recursos vegetales apícolas que aportan para la composición de los productos originados en los apiarios, dado que adquieren las propiedades de las plantas que lo producen, es decir, origen floral y geográfico; lo cual genera la posibilidad de obtener productos apícolas con características únicas (Montenegro, 2013). Al constituir una actividad económica en crecimiento que representa un potencial de riqueza por los diversos beneficios que se pueden obtener a través del aprovechamiento artesanal o industrial. En este trabajo se estudian las plantas de interés apícola que integran áreas adyacentes a dos colmenares, cuyas unidades productivas poseen sistemas agroecológicos, que contribuyen al paisaje, a la protección del equilibrio del ambiente y a la producción agroforestal (Guallpa et al., 2019a).

La caracterización de la flora melífera es muy necesaria para el manejo de este valioso recurso en aras de promover el desarrollo de la apicultura en zonas de trópico. Basándose en el monitoreo de las visitas de abejas a las plantas apícolas en floración (Guallpa et al., 2020). Teniendo en cuenta que es uno de los pilares fundamentales que aporta al manejo de los colmenares una vez que se integran para la gestión de un apiario, elementos como las abejas, tecnología y los apicultores, son claves en esta actividad agropecuaria para definir la alternativa productiva (Sánchez, 2013). Por ello, se planteó identificar especies vegetales con potencial apícola, y estimar su abundancia más la durabilidad de la floración en las dos áreas de investigación.

\section{Metodología}

Se establecieron como sitios de muestreo dos predios que albergan dos colmenares, mismos que están conformados por múltiples sistemas de uso de la tierra que integran las dos unidades apícolas privadas, el colmenar C1, ubicado en el kilómetro 38 vía Santo Domingo-Quinindé, Cantón La Concordia, Provincia de Santo Domingo de los Tsáchilas. El colmenar C2, se localiza en el km 7 1/2 de la Vía Quevedo-El Empalme, Cantón Mocache, Provincia de Los Ríos. Ambos lugares están dentro de la formación: Bosque húmedo tropical (Sierra et al., 1999).

Geográficamente se localizan en los puntos de referencia: UTM Zona 17S Datum WGS 84, y los principales parámetros climáticos de cada sitio se muestran en la tabla 1. 
Tabla 1

Localización geográfica y parámetros climáticos de los dos lugares de los colmenares evaluados

\begin{tabular}{|c|c|c|c|c|c|c|}
\hline Lugares & Longitud & Latitud & $\begin{array}{l}\text { Altitud } \\
\text { (msnm) }\end{array}$ & $\begin{array}{l}\text { Temperatura } \\
{ }^{\circ} \mathrm{C}\end{array}$ & $\begin{array}{l}\text { Precipitación } \\
\mathrm{mm}\end{array}$ & $\begin{array}{c}\text { Humedad } \\
\text { relativa } \\
\%\end{array}$ \\
\hline $\mathrm{La}$ & - & - & $240 \mathrm{a}$ & 25,5 & 3340,9 & 87 \\
\hline $\begin{array}{l}\text { Concordia } \\
\text { (C1) }\end{array}$ & $79.3735061^{\circ}$ & $0.0232177^{\circ}$ & 315 & & & \\
\hline $\begin{array}{l}\text { Mocache } \\
\text { (C2) }\end{array}$ & $\begin{array}{l}-79.4986275 \\
\circ\end{array}$ & $\begin{array}{l}- \\
1.0855355^{\circ}\end{array}$ & 90 & 24,7 & $1250-2000$ & 85 \\
\hline
\end{tabular}

\section{Métodos}

En esta caracterización se aplicó los métodos: documental y de campo, tanto para el reconocimiento de especies vegetales melíferas, su cantidad y durabilidad de la floración de aquellas plantas apartadas entre 800 a 1000 m hasta cada colmenar (García de la Figal, 2018; Calvache, 2016). La valoración de plantas apícolas se procedió a monitorear durante el año 2020. Por su parte, la abundancia y floridez se valoró cada 30 días, según planificación para esta investigación, con la ejecución de las siguientes actividades:

\section{Reconocimiento de los usos de la tierra de cada colmenar}

Se realizó mediante dos recorridos por cada una de las subáreas que conforman las dos unidades productivas, donde se efectuó el registro de los usos de la tierra que caracterizan a cada predio para su categorización, con se muestra en la tabla 2, al utilizar el guion de observación directa (Guallpa et al., 2019b).

\section{Tabla 2}

Categorías de arreglos de la vegetación utilizados para la caracterización de la flora apícola

\begin{tabular}{ll}
\hline \multicolumn{1}{c}{ Categoría } & \multicolumn{1}{c}{ Especificaciones } \\
\hline Bosque & $\begin{array}{l}\text { Ecosistema arbóreo, que se caracteriza por la } \\
\text { presencia de árboles de diferentes especies nativas, } \\
\text { edades y portes variados, con uno o más estratos. }\end{array}$ \\
\hline Plantación & $\begin{array}{l}\text { Especies foráneas de rápido crecimiento, } \\
\text { plantadas para la producción, comercialización o } \\
\text { conservación }\end{array}$ \\
\hline Arbustos & $\begin{array}{l}\text { Vegetación arbustiva con menos de } 5 \text { m de altura } \\
\text { que se encuentra formando congregados de } \\
\text { especies vegetales o no }\end{array}$ \\
\hline
\end{tabular}


Tabla 2

Categorías de arreglos de la vegetación utilizados para la caracterización de la flora apícola (continuación)

\begin{tabular}{|c|c|}
\hline Categoría & Especificaciones \\
\hline Rastrojo & $\begin{array}{l}\text { Vegetación herbácea o leñosa que nace por } \\
\text { regeneración natural. De poca altura, máximo } 2 \\
\text { metros }\end{array}$ \\
\hline Pastos & Pasturas naturales o plantadas \\
\hline Cultivos & $\begin{array}{l}\text { Vegetación compuesta por especies de uso } \\
\text { agrícola. Los cultivos pueden ser permanentes o } \\
\text { temporales }\end{array}$ \\
\hline $\begin{array}{l}\text { Sistemas } \\
\text { agroforestales }\end{array}$ & $\begin{array}{l}\text { Conjunto de diferentes especies de plantas con } \\
\text { fines específicos que constituyen arreglos. Un } \\
\text { ejemplo son las huertas o sistemas agroforestales. }\end{array}$ \\
\hline Bordes de caminos & $\begin{array}{l}\text { Aquellos márgenes adyacentes a las vías provistas } \\
\text { de vegetación establecida o no }\end{array}$ \\
\hline Ornamental & $\begin{array}{l}\text { Plantas que se cultivan con una finalidad estética para } \\
\text { embellecer un determinado espacio }\end{array}$ \\
\hline
\end{tabular}

Fuente: Guion de observación directa aplicada (Guallpa et al., 2019b)

Monitoreo e identificación taxonómica de especies vegetales

Se aplicó el inventario por muestreo a las áreas con diferentes usos de la tierra en cada predio, donde se identificó los elementos florísticos con potencial apícola que integran los diferentes modelos de plantación adyacentes a los dos sistemas productivos. Mediante tres visitas a cada predio, al distinguir aquellas plantas que son frecuentadas por las abejas dada la oferta de néctar y/o polen, por medio del reconocimiento de ciertos especímenes en campo a partir de sus características diagnósticas con el uso de binoculares y otros con muestras herborizadas fueron identificados en el Herbario ESPOCH (Caranqui et al., 2021).

Verificación de la oferta de néctar o polen de las especies vegetales

Se registró la flora melífera, al observar el estado de floración, y la forma de elegir por las abejas; el néctar, polen o la combinación néctar y polen con el uso del protocolo de monitoreo de esos recursos con la aplicación del esquema de Silva \& Restrepo (2012).

\section{Cuantificación de la abundancia de la flora melífera}

Se usó la categorización semi - cuantitativa diseñada por May \& Rodríguez (2012), quienes en su investigación fijaron valores entre 1 a 5 , para calcular la abundancia, con la serie que se indica en la tabla 3. 
Tabla 3

Escala para la cuantificación de la abundancia flora apícola (A)

\begin{tabular}{cl}
\hline Grado & \multicolumn{1}{c}{ Pormenorización } \\
\hline 1 & $\begin{array}{l}\text { Menos de } 1000 \text { individuos con floridez dentro del perímetro de } \\
\text { actividad de las abejas, cubriendo menos de 1\% de la superficie a } \\
\text { valorar }\end{array}$ \\
\hline 2 & $\begin{array}{l}\text { Fluctúa de 1000 a 10000 individuos con floridez dentro del perímetro } \\
\text { de actividad de las abejas, cubriendo menos de 1\% de la superficie }\end{array}$ \\
\hline 3 & $\begin{array}{l}\text { Mayor a 10000 individuos con floridez dentro del perímetro de } \\
\text { actividad de las abejas cubriendo menos de 1\% de la superficie }\end{array}$ \\
\hline 4 & $\begin{array}{l}\text { Individuos con floridez dentro del perímetro de actividad de las } \\
\text { abejas cubren entre 1\% y 5\% de la superficie }\end{array}$ \\
\hline 5 & $\begin{array}{l}\text { Individuos con floridez dentro del perímetro de actividad de las } \\
\text { abejas cubren más de 5\% de la superficie }\end{array}$ \\
\hline
\end{tabular}

Fuente: May \& Rodríguez (2012)

Finalmente, se establecen valores de 1 a 5 para representar la durabilidad de floración de cada una de las especies melíferas (DF; tabla 4). Mediante la revisión de la floridez en la base de datos especializada Trópicos.Org. Missouri Botanical Garden (2021) más el monitoreo en campo cada 30 días.

Tabla 4

Escala del índice durabilidad de la floración (DF)

\begin{tabular}{cl}
\hline Grado & \multicolumn{1}{c}{ Pormenorización } \\
\hline 1 & De un mes o menos \\
2 & Entre 2 o 3 meses \\
3 & Entre 4 o 5 meses \\
4 & Entre 6 o 7 meses \\
5 & Entre $8-12$ meses \\
\hline
\end{tabular}

Fuente: May \& Rodríguez (2012)

Sistematización de la información

Para el registro, procesamiento, y análisis de datos de plantas melíferas, su abundancia y durabilidad de la floración, se utilizó la hoja de cálculo de Excel.

\section{Análisis estadístico}

Se probó el comportamiento normal de las variables, abundancia (A) y durabilidad de la floración (DF) con el estadígrafo Shapiro-Wilk; al verificar que no se cumple el supuesto de normalidad para dos muestras independientes, se aplicó la prueba de Mann Whitney en el programa estadístico SPSS Statistics 24 (Quezada, 2017). 
www.cienciadigital.org

\section{Resultados}

\section{A. Especies apícolas identificadas en los colmenares $C 1$ y $C 2$}

Las especies florísticas que se identificaron para el Colmenar 1 ubicado dentro del cantón La Concordia (tabla 5) son 14 especies, pertenecientes a 12 familias. De las 14 especies de plantas, todas fueron identificadas a nivel de especie. Según su hábito de crecimiento se distribuyen en 9 especies arbóreas, 4 especies de crecimiento arbustivo y una especie del tipo arbustivo/arbóreo. En referencia al recurso ofertado; 2 plantas melíferas corresponden a productoras de néctar, mientras que 13 proveen néctar y polen.

\section{Tabla 5}

Especies vegetales apícolas adyacentes a los dos colmenares

\section{Colmenar 1}

\begin{tabular}{|c|c|c|c|c|}
\hline No & Familia & Nombre científico & Hábito de crecimiento & $\begin{array}{l}\text { Recurso } \\
\text { ofertado }\end{array}$ \\
\hline 1 & Bignoniaceae & $\begin{array}{l}\text { Tabebuia chrysantha } \\
\text { (Jacq.) G. Nicholson }\end{array}$ & Arbóreo & $\mathrm{N} / \mathrm{P}$ \\
\hline 2 & Boraginaceae & $\begin{array}{l}\text { Cordia alliodora (Ruiz \& } \\
\text { Pav.) Oken }\end{array}$ & Arbóreo & $\mathrm{N} / \mathrm{P}$ \\
\hline 3 & Euphorbiaceae & $\begin{array}{l}\text { Hevea brasiliensis (Willd. } \\
\text { ex A. Juss.) Müll. Arg. }\end{array}$ & Arbóreo & $\mathrm{N}$ \\
\hline 4 & Fabaceae & $\begin{array}{l}\text { Erythrina edulis Triana ex } \\
\text { Michell. }\end{array}$ & Arbóreo & $\mathrm{N} / \mathrm{P}$ \\
\hline 5 & Fabaceae & Inga edulis Mart. & Arbóreo & $\mathrm{N} / \mathrm{P}$ \\
\hline 6 & Fabaceae & Senna alata (L.) Roxb. & Arbóreo & $\mathrm{N} / \mathrm{P}$ \\
\hline 7 & Lauraceae & Persea americana Mill & Arbóreo & $\mathrm{N} / \mathrm{P}$ \\
\hline 8 & Malvaceae & Hibiscus rosa-sinensis $\mathrm{L}$ & Arbustivo & $\mathrm{N} / \mathrm{P}$ \\
\hline 9 & $\begin{array}{l}\text { Melastomatacea } \\
\mathrm{e}\end{array}$ & $\begin{array}{l}\text { Tibouchina mollis (Bonpl. } \\
\text { ) Cogn. }\end{array}$ & Arbustivo & $\mathrm{N} / \mathrm{P}$ \\
\hline 10 & Nyctaginaceae & $\begin{array}{l}\text { Bougainvillea spectabilis } \\
\text { Willd. }\end{array}$ & Arbustivo & $\mathrm{N} / \mathrm{P}$ \\
\hline 11 & Piperaceae & Piper aduncum L. & Arbustivo & $\mathrm{N}$ \\
\hline 12 & Polygonaceae & $\begin{array}{l}\text { Triplaris cumingiana } \\
\text { Fisch. \& C.A. Mey. }\end{array}$ & Arbóreo & $\mathrm{N} / \mathrm{P}$ \\
\hline 13 & Rutaceae & Citrus reticulata Blanco & Arbóreo & $\mathrm{N} / \mathrm{P}$ \\
\hline 14 & Solanaceae & $\begin{array}{l}\text { Cestrum racemosum Ruíz } \\
\& \text { Pav. }\end{array}$ & Arbustivo/arbóreo & $\mathrm{N} / \mathrm{P}$ \\
\hline
\end{tabular}




\section{Tabla 5}

Especies vegetales apícolas adyacentes a los dos colmenares (continuación)

\section{Colmenar 2}

\begin{tabular}{|c|c|c|c|c|}
\hline $\mathrm{N}^{\circ}$ & Familia & Nombre científico & $\begin{array}{c}\text { Hábito de } \\
\text { crecimiento }\end{array}$ & $\begin{array}{l}\text { Recurso } \\
\text { ofertado }\end{array}$ \\
\hline 1 & Anacardiaceae & Mangifera indica $\mathrm{L}$ & Arbóreo & $\mathrm{P}$ \\
\hline 2 & Anacardiaceae & Spondias purpurea $\mathrm{L}$ & Arbóreo & $\mathrm{N}$ \\
\hline 3 & Anacardiaceae & Anacardium occidentale $\mathrm{L}$ & Arbóreo & $\mathrm{N} / \mathrm{P}$ \\
\hline 4 & Arecaceae & Attalea insignis (Mart.) Dr. & Palmera & $\mathrm{N} / \mathrm{P}$ \\
\hline 5 & Asteraceae & $\begin{array}{l}\text { Vernonanthura patens (Kunth) H. } \\
\text { Rob. }\end{array}$ & Arbustivo & $\mathrm{N} / \mathrm{P}$ \\
\hline 6 & Asteraceae & Tridax procumbens $\mathrm{L}$ & $\begin{array}{l}\text { Herbáceo } \\
\text { perenne }\end{array}$ & $\mathrm{N} / \mathrm{P}$ \\
\hline 7 & Bixaceae & $\begin{array}{l}\text { Cochlospermum vitifolium (Willd.) } \\
\text { Spreng. }\end{array}$ & Arbóreo & $\mathrm{N} / \mathrm{P}$ \\
\hline 8 & Boraginaceae & $\begin{array}{l}\text { Cordia alliodora (Ruiz \& Pav.) } \\
\text { Oken }\end{array}$ & Arbóreo & $\mathrm{N} / \mathrm{P}$ \\
\hline 9 & Capparaceae & Capparis scabrida Kunth & Arbustivo & $\mathrm{N} / \mathrm{P}$ \\
\hline 10 & Capparaceae & Capparis crotonoides Kunth & Arbóreo & $\mathrm{N} / \mathrm{P}$ \\
\hline 11 & Cucurbitaceae & Momordica charantia A. Rich. & Mata & $\mathrm{N}$ \\
\hline 12 & Fabaceae & $\begin{array}{l}\text { Erythrina poeppigiana (Walp.) O.F. } \\
\text { Cook }\end{array}$ & Arbóreo & $\mathrm{N} / \mathrm{P}$ \\
\hline 13 & Fabaceae & Albizia guachapele (Kunth) Dugand & Arbóreo & $\mathrm{N} / \mathrm{P}$ \\
\hline 14 & Fabaceae & Inga spectabilis (Vahl) Willd. & Arbóreo & $\mathrm{N} / \mathrm{P}$ \\
\hline 15 & Fabaceae & Samanea saman (Jacq.) Merr. & Arbóreo & $\mathrm{N} / \mathrm{P}$ \\
\hline 16 & Fabaceae & Glycine $\max (\mathrm{L}$.$) Merr.$ & Herbáceo & $\mathrm{N} / \mathrm{P}$ \\
\hline 17 & Geraniaceae & Pelargonium $s p$ & Herbáceo & $\mathrm{N} / \mathrm{P}$ \\
\hline 18 & Hydrangeaceae & Hydrangea $s p$ & Herbáceo & $\mathrm{N} / \mathrm{P}$ \\
\hline 19 & Lamiaceae & Gmelina arborea Roxb. & Arbóreo & $\mathrm{N}$ \\
\hline 20 & Malvaceae & Corchorus hirtus L & $\begin{array}{l}\text { Herbáceo/su } \\
\text { barbustivo }\end{array}$ & N/P \\
\hline 21 & Poaceae & Cynodon dactylon (L.) Pers. & Herbáceo & $\mathrm{N}$ \\
\hline 22 & Solanaceae & Nicotiana glauca Graham. & Arbustivo & $\mathrm{N} / \mathrm{P}$ \\
\hline
\end{tabular}

Para el colmenar 2 situado dentro del Cantón Mocache, las especies vegetales reconocidas son 22 especies, pertenecientes a 14 familias. Del total de especies de plantas, 20 fueron identificadas a nivel de especie y 2 a nivel de género. Según su hábito de crecimiento se distribuyen en 11 especies arbóreas, una de tipo palmera, 3 especies de crecimiento arbustivo, 4 especies de tipo herbáceo; una especie herbáceo perenne, otra herbácea /subarbustiva y una especie de tipo mata. En referencia al recurso ofertado se reporta a 4 plantas productoras de néctar, una de polen y 19 ofertan tanto néctar como polen (tabla 5). 
Los datos obtenidos son fundamentales al contar con información de las principales familias botánicas identificadas para los sitios de estudio con especies de potencial nectarífero-polinífero para el colmenar C1 se destaca la familia Fabaceae y en el caso del colmenar 2 predomina la Familia Fabaceae, seguida de Anacardiaceae, Asteraceae y Capparaceae. Resultados que coinciden de forma parcial en relación con las familias Fabaceae y Asteraceae, porque sus especies vegetales les proporcionan en la mayoría de los casos, polen y néctar (Pinilla y Nates, 2015). De forma específica el néctar extra floral producido por $H$. brasiliensis es un recurso apícola importante en las regiones tropicales, raramente se encuentra polen de $H$. brasiliensis en los análisis microscópicos. No obstante, que la miel de la especie indicada contiene una amplia diversidad de tipos de polen (Zavala, 2013).

\section{B. Abundancia de especies vegetales en los colmenares $C 1$ y $C 2$}

La valoración de la cantidad de individuos por especie vegetal determinó la predominancia de individuos con floridez de Glycine max ocupando entre $1 \%$ y $5 \%$ de la superficie de cultivos del colmenar $\mathrm{C} 2$, seguida de las especies forestales con más de 10.000 individuos con flores de Cordia alliodora en ambos colmenares y Gmelina arborea en el C2 integrando plantaciones con fines madereros. En menor cantidad de individuos de hábito de crecimiento herbáceo entre 1.000 a 10.000 individuos de Corchorus hirtus en la superficie de restrojo y Cynodon dactylon en la superficie de pastos del C2. Por su parte también entre 1.000 a 10.000 individuos de Triplaris cumingiana, especie maderable ocupando el área de sistemas agroforestales del colmenar C1. El resto de las especies vegetales con una ocupación de menos de 1.000 individuos con flores presentes (Tabla 6).

Tabla 6

Abundancia de flora melífera para los colmenares C1 y C2

\begin{tabular}{|c|c|c|c|c|c|c|c|c|c|c|c|c|c|c|c|c|}
\hline \multirow[t]{3}{*}{ Nombre científico } & \multicolumn{2}{|c|}{ 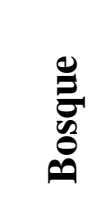 } & \multicolumn{2}{|c|}{ 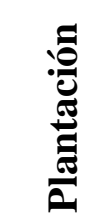 } & \multicolumn{2}{|c|}{ 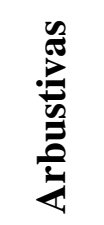 } & \multicolumn{2}{|c|}{ 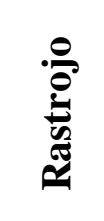 } & \multicolumn{2}{|c|}{ 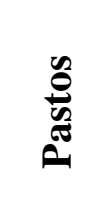 } & \multicolumn{2}{|c|}{ 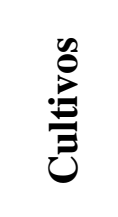 } & \multicolumn{2}{|c|}{ 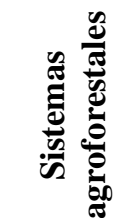 } & \multicolumn{2}{|c|}{ 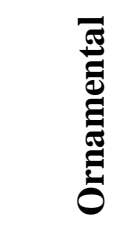 } \\
\hline & $\mathrm{C}$ & $\mathrm{C}$ & $\mathrm{C}$ & $\mathrm{C}$ & $\mathrm{C}$ & $\mathrm{C}$ & $\mathrm{C}$ & $\mathrm{C}$ & $\mathrm{C}$ & $\mathrm{C}$ & $\mathrm{C}$ & $\mathrm{C}$ & $\mathrm{C}$ & $\mathrm{C}$ & $\mathrm{C}$ & $\mathrm{C}$ \\
\hline & 1 & 2 & 1 & 2 & 1 & 2 & 1 & 2 & 1 & 2 & 1 & 2 & 1 & 2 & 1 & 2 \\
\hline Mangifera indica & & & & & & & & & & & & & & 1 & & \\
\hline Spondias purpurea & & 1 & & & & & & & & & & & & & & \\
\hline $\begin{array}{l}\text { Anacardium } \\
\text { occidentale }\end{array}$ & & & & & & & & & & & & & & 1 & & \\
\hline Attalea insignis & & & & & & & & & & & & & & 1 & & \\
\hline $\begin{array}{l}\text { Vernonanthura } \\
\text { patens }\end{array}$ & & & & & & & & & & & & & & 1 & & \\
\hline
\end{tabular}




\section{ISSN: 2773-7330}

Vol. 4 No. 1, pp. 28 - 45 , enero - marzo 2022

www.cienciadigital.org

Tabla 6

Abundancia de flora melífera para los colmenares C1 y C2 (continuación)

\begin{tabular}{|c|c|c|c|c|c|c|c|c|c|c|c|c|c|c|c|c|}
\hline \multirow[t]{3}{*}{ Nombre científico } & \multicolumn{2}{|c|}{ 莺 } & \multicolumn{2}{|c|}{ 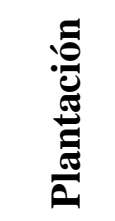 } & \multicolumn{2}{|c|}{ 音 } & \multicolumn{2}{|c|}{ 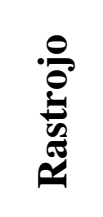 } & \multicolumn{2}{|c|}{ 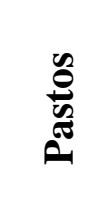 } & \multicolumn{2}{|c|}{ 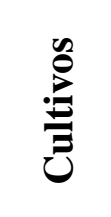 } & \multicolumn{2}{|c|}{ 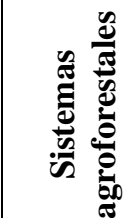 } & \multicolumn{2}{|c|}{ 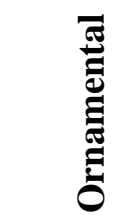 } \\
\hline & $\mathrm{C}$ & $\mathrm{C}$ & $\mathrm{C}$ & $\mathrm{C}$ & $\mathrm{C}$ & $\mathrm{C}$ & $\mathrm{C}$ & $\mathrm{C}$ & $\mathrm{C}$ & $\mathrm{C}$ & $\mathrm{C}$ & $\mathrm{C}$ & $\mathrm{C}$ & $\mathrm{C}$ & $\mathrm{C}$ & $\mathrm{C}$ \\
\hline & 1 & 2 & 1 & 2 & 1 & 2 & 1 & 2 & 1 & 2 & 1 & 2 & 1 & 2 & 1 & 2 \\
\hline Tridax procumbens & & & & & & & & 1 & & & & & & & & \\
\hline $\begin{array}{l}\text { Cochlospermum } \\
\text { vitifolium }\end{array}$ & & & & & & & & & & & & & & & & 1 \\
\hline Cordia alliodora & & & 3 & 3 & & & & & & & & & & & & \\
\hline Tabebuia chrysantha & & & & & & & & & & & & & & & 1 & \\
\hline Capparis scabrida & & & & & & & & & & & & & & 1 & & \\
\hline Capparis crotonoides & & & & & & & & & & & & & & 1 & & \\
\hline Momordica charantia & & & & & & & & 1 & & & & & & & & \\
\hline Hevea brasiliensis & & & 1 & & & & & & & & & & & & & \\
\hline Erythrina poeppigiana & & 1 & & & & & & & & & & & & & & \\
\hline Erythrina edulis & & & & & & & & & & & & & 1 & & & \\
\hline Albizia guachapele & & 1 & & & & & & & & & & & & & & \\
\hline Inga spectabilis & & 1 & & & & & & & & & & & & & & \\
\hline Inga edulis & & & & & & & & & & & & & & & 1 & \\
\hline Senna alata & & & & & & & & & & & & & & & 1 & \\
\hline Samanea saman & & & & & & & & & & & & & & 1 & & \\
\hline Glycine $\max$ & & & & & & & & & & & & 4 & & & & \\
\hline Pelargonium sp & & & & & & & & & & & & & & & & 1 \\
\hline Hydrangea sp & & & & & & & & & & & & & & & & 1 \\
\hline Persea americana & & & & & & & & & & & 1 & & & & & \\
\hline Gmelina arborea & & & & 3 & & & & & & & & & & & & \\
\hline Corchorus hirtus & & & & & & & & 2 & & & & & & & & \\
\hline Hibiscus rosa-sinensis & & & & & & 1 & & & & & & & & & & \\
\hline Tibouchina mollis & & & & & & & & & & & & & 1 & & & \\
\hline $\begin{array}{l}\text { Bougainvillea } \\
\text { spectabilis }\end{array}$ & & & & & & & & & & & & & & & 1 & \\
\hline Piper aduncum & & & & & & & & & & & & & & & 1 & \\
\hline Cynodon dactylon & & & & & & & & & & 2 & & & & & & \\
\hline Triplaris cumingiana & & & & & & & & & & & & & 2 & & & \\
\hline Citrus reticulata & & & & & & & & & & & 1 & & & & & \\
\hline
\end{tabular}




\section{Tabla 6}

Abundancia de flora melífera para los colmenares C1 y C2 (continuación)

\begin{tabular}{|c|c|c|c|c|c|c|c|c|c|c|c|c|c|c|c|c|}
\hline \multirow[t]{2}{*}{$\begin{array}{l}\text { Nombre } \\
\text { científico }\end{array}$} & \multicolumn{2}{|c|}{ 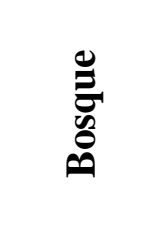 } & \multicolumn{2}{|c|}{ 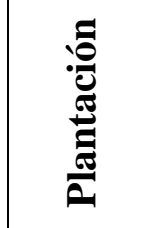 } & \multicolumn{2}{|c|}{ 总 } & \multicolumn{2}{|c|}{ 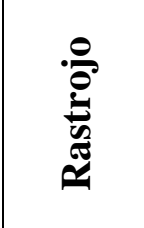 } & \multicolumn{2}{|c|}{$\begin{array}{l}\tilde{\theta} \\
\tilde{\mathscr{\theta}} \\
\tilde{\theta}\end{array}$} & \multicolumn{2}{|c|}{ 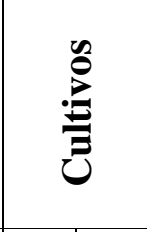 } & \multicolumn{2}{|c|}{ 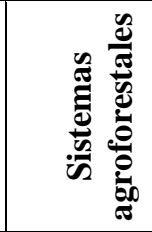 } & \multicolumn{2}{|r|}{ 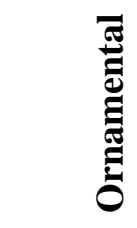 } \\
\hline & $\mathrm{C} 1$ & $\mathrm{C} 2$ & C1 & $\mathrm{C} 2$ & C1 & $\mathrm{C} 2$ & C1 & $\mathrm{C} 2$ & C1 & $\mathrm{C} 2$ & C1 & $\mathrm{C} 2$ & C1 & $\mathrm{C} 2$ & C1 & $\mathrm{C} 2$ \\
\hline $\begin{array}{l}\text { Cestrum } \\
\text { racemosum }\end{array}$ & 1 & & & & & & & & & & & & & & & \\
\hline $\begin{array}{l}\text { Nicotiana } \\
\text { glauca }\end{array}$ & & & & & & 1 & & & & & & & & & & \\
\hline
\end{tabular}

Abundancia: (1) Menos de 1000 individuos con floridez dentro del perímetro de actividad de las abejas, cubriendo menos de $1 \%$ de la superficie (2) Fluctúa de 1000 a 10000 individuos con floridez dentro del perímetro de actividad de las abejas, cubriendo menos de $1 \%$ de la superficie (3) Mayor a 10000 individuos con floridez dentro del perímetro de actividad de las abejas y (4) Individuos con floridez dentro del perímetro de actividad de las abejas cubren más de $5 \%$ de la superficie.

Como puede apreciarse el estadígrafo de U de Mann-Whitney fue de 140,000 y el valor de p (Sig. asintót. bilateral) es 0,510 por lo que se acepta la hipótesis nula y se concluye que el nivel de abundancia de especies apícolas no difiere entre los colmenares $\mathrm{C} 1$ y C2, con un nivel de significación del 5\%, como se muestra en la tabla 7.

\section{Tabla 7}

Estadísticos de contraste ${ }^{a}$

\begin{tabular}{lr}
\hline & \multicolumn{1}{c}{ Abundancia } \\
\hline U de Mann-Whitney & 140,000 \\
$Z$ &,- 658 \\
Sig. asintót. (bilateral) &, 510 \\
Sig. exacta [2*(Sig. &, $665^{\mathrm{b}}$ \\
\hline
\end{tabular}

a. Variable de agrupación: Colmenares

b. No corregidos para los empates

De acuerdo a lo expuesto, aunque sin diferir desde el punto de vista estadístico para la cantidad de plantas melíferas entre los colmenares $\mathrm{C} 1$ y $\mathrm{C} 2$, de forma numérica la mayor cantidad de individuos de especies con potencial melífero en los dos colmenares, se tiene con la especie herbácea Glycine max que ocupa el área de cultivos del colmenar C2, luego se tiene a individuos de las especies maderables Cordia alliodora para ambos colmenares, y Gmelina arborea del colmenar C2, situación que ocurre dado que son predios que dentro de su gestión están formados por cultivos agrícolas por una parte y por otra la 
existencia de plantaciones forestales, así la presencia de áreas de bosques, existencia de sistemas agroforestales, parcelas de pastos y ciertas áreas de uso ornamental, son atributos que inciden en el aporte de recursos apícolas, donde las abejas realizan su aprovechamiento según su gusto, preferencia y accesibilidad, desplazándose a diferentes distancias, direcciones y coberturas vegetales que están disponibles de acuerdo al manejo técnico de cada predio y los objetivos propuestos de cada propietario (Nates, 2016).

\section{Durabilidad de floración de especies vegetales en los colmenares C1 y C2}

En el colmenar C1 se encontró una mayor floración existen 8 especies que florecen entre 10 a 12 meses y con el menor valor se presenta Hevea brasiliensis cuya floración ocurre entre los meses de septiembre y octubre lo que muestra la disponibilidad de estas para la alimentación de las abejas. En el caso del colmenar C2 a pesar de haber identificado una mayor cantidad de especies vegetales, solo tres florecen durante todo el año, seis entre 4 a 6 meses, once entre 2 a 3 meses, y dos especies florecen específicamente el mes de diciembre. Lo que indica una menor duración de la floración de especies vegetales circundantes al Colmenar $\mathrm{C} 1$, lo que incide en la oferta de polen y néctar para el aprovechamiento por parte de Apis mellifera, como se muestra en la tabla 8.

\section{Tabla 8}

Duración de la floración de especies melíferas adyacentes a los colmenares C1 y C2

\begin{tabular}{|c|c|c|c|c|c|c|c|c|c|c|c|c|c|}
\hline \multicolumn{14}{|c|}{ Colmenar 1} \\
\hline Nombre científico & $\mathbf{E}$ & $\mathbf{F}$ & M & $\mathbf{A}$ & $\mathbf{M}$ & $\mathbf{J}$ & $\mathbf{J}$ & $\mathbf{A}$ & $\mathbf{S}$ & $\mathbf{O}$ & $\mathbf{N}$ & D & DF \\
\hline Tabebuia chrysantha & & & & & & & & & & & & & 3 \\
\hline Cordia alliodora & & & & & & & & & & & & & 5 \\
\hline Hevea brasiliensis & & & & & & & & & & & & & 2 \\
\hline Erythrina edulis & & & & & & & & & & & & & 5 \\
\hline Senna alata & & & & & & & & & & & & & 5 \\
\hline Inga edulis & & & & & & & & & & & & & 5 \\
\hline Persea americana & & & & & & & & & & & & & 5 \\
\hline Hibiscus rosa-sinensis & & & & & & & & & & & & & 5 \\
\hline Tibouchina mollis & & & & & & & & & & & & & 2 \\
\hline Bougainvillea spectabilis & & & & & & & & & & & & & 3 \\
\hline Piper aduncum & & & & & & & & & & & & & 5 \\
\hline Triplaris cumingiana & & & & & & & & & & & & & 2 \\
\hline Citrus reticulata & & & & & & & & & & & & & 2 \\
\hline Cestrum racemosum & & & & & & & & & & & & & 5 \\
\hline \multicolumn{14}{|c|}{ Colmenar 2} \\
\hline Mangifera indica & & & & & & & & & & & & & 1 \\
\hline Spondias purpurea & & & & & & & & & & & & & 2 \\
\hline
\end{tabular}




\section{Tabla 8}

Duración de la floración de especies melíferas adyacentes a los colmenares C1 y C2 (continuación)

\begin{tabular}{|l|l|l|l|l|l|l|l|l|l|l|l|c|c|}
\hline \multicolumn{9}{|c|}{ Colmenar 2 } \\
\hline Nombre científico & E & F & M & A & M & J & J & A & S & O & N & D & DF \\
\hline Anacardium occidentale & & & & & & & & & & & & & 3 \\
\hline Attalea insignis & & & & & & & & & & & & & 4 \\
\hline Vernonanthura patens & & & & & & & & & & & & & 1 \\
\hline Tridax procumbens & & & & & & & & & & & & 5 \\
\hline Cochlospermum vitifolium & & & & & & & & & & & & & 2 \\
\hline Cordia alliodora & & & & & & & & & & & \\
\hline Capparis scabrida & & & & & & & & & & & \\
\hline Capparis crotonoides & & & & & & & & & & & & \\
\hline Momordica charantia & & & & & & & & & & & & \\
\hline Erythrina poeppigiana & & & & & & & & & & & & \\
\hline Albizia guachapele & & & & & & & & & & & & \\
\hline Inga spectabilis & & & & & & & & & & & & 2 \\
\hline Samanea saman & & & & & & & & & & & & & 3 \\
\hline Glycine max & & & & & & & & & & & & & 2 \\
\hline Pelargonium sp & & & & & & & & & & & & & 5 \\
\hline Hydrangea sp & & & & & & & & & & & & 5 \\
\hline Gmelina arborea & & & & & & & & & & & & & 2 \\
\hline Corchorus hirtus & & & & & & & & & & & & 2 \\
\hline Cynodon dactylon & & & & & & & & & & & & 1 \\
\hline Nicotiana glauca & & & & & & & & & & & & 3 \\
\hline
\end{tabular}

De acuerdo con el estadígrafo de U de Mann-Whitney que fue de 84,000 y el valor de $\mathrm{p}$ (Sig. asintót. (bilateral)) es 0,017 por lo que se rechaza la hipótesis nula y se concluye que la duración de la floración de las especies apícolas difiere entre los colmenares C1 y C2, con un nivel de significación del 5\%, como se muestra en la tabla 9.

Tabla 9

Estadísticos de contraste ${ }^{a}$

\begin{tabular}{lr}
\hline & \multicolumn{2}{c}{$\begin{array}{c}\text { Duración de la } \\
\text { Floración }\end{array}$} \\
\hline U de Mann-Whitney & 84,000 \\
$Z$ & $-2,377$ \\
Sig. asintót. (bilateral) &, 017 \\
Sig. exacta [2*(Sig. &, $023^{\mathrm{b}}$ \\
unilateral)] & \\
\hline
\end{tabular}

a. Variable de agrupación: Colmenares

b. No corregidos para los empates 
La duración de la florescencia de las especies vegetales que ofertan alimento a las abejas de los colmenares $\mathrm{C} 1$ y C2 fluctúa entre 1 a 12 meses variando en función del espécimen y la cantidad de individuos que conforman los diferentes sistemas de uso del suelo, su etapa de crecimiento al momento de ser evaluadas, la gestión de cada subárea dentro de cada predio más la influencia de los factores edafoclimáticos que influyen en el proceso de fenología de ciertas especies vegetales de un determinado sitio (Guallpa et al., 2020).

\section{Conclusiones}

- De forma inicial se ha identificado que las principales familias botánicas con especies nectaríferas y poliníferas en los sitios de estudio son; Fabaceae, Anacardiaceae, Asteraceae y Capparaceae. Cuyo listado de especies vegetales con potencial melífero afines a las abejas para los sitios donde se encuentran los dos apiarios en esta investigación es preliminar, y es fundamental ampliar la identificación botánica, así como la colecta y el análisis de las muestras de polen para lograr la confirmación de los diferentes tipos de polen que caracterizan a las mieles que se producen en los dos apiarios y de otros localizados en los mismos cantones o diferentes de la región Costa de condiciones técnicas similares.

- La oferta del recurso floral tiene un comportamiento similar referente a la cantidad de plantas melíferas entre los colmenares $\mathrm{C} 1$ y $\mathrm{C} 2$ a pesar de poseer mayor cantidad de especies con potencial apícola el colmenar C2, lo cual obedece al manejo que se aplica a las áreas que integran los diferentes sistemas de uso del suelo que posee cada predio.

- La duración de la florescencia de las especies con potencial melífero que ofertan alimento a las abejas de los colmenares $\mathrm{C} 1$ y $\mathrm{C} 2$ se registra entre 1 a 12 meses para el año 2020 con predominancia para el colmenar C1 de Cordia alliodora, Erythrina edulis, Senna alata, Inga edulis, Persea americana, Hibiscus rosasinensis, Piper aduncum, y Cestrum racemosum que florecen entre 10 a 12 meses y con el menor valor se presenta Hevea brasiliensis cuya floración ocurre entre los meses de septiembre y octubre. En cambio, en el colmenar C1 solo Tridax procumbens, Pelargonium sp, y Hydrangea sp florecen durante todo el año y con el menor valor a Mangifera indica y Cynodon dactylon que florecen específicamente el mes de diciembre.

\section{Referencias bibliográficas}

Agrocalidad. (2015). Guía de Buenas Prácticas Apícolas. Inocuidad de los Alimentos. Resolución N0053. http://extwprlegs1.fao.org/docs/pdf/ecu167496.pdf 
Calvache, J. (2016). La investigación científica como alternativa en la formación profesional. CEPUN.

Caranqui, J., Guilcapi E., Espinoza, V., y Ortiz, L. (2021). Fenología reproductiva en base de datos de herbarios de Vaccinium floribundum Kunth (Ericaceae), Ecuador. Dominio de las Ciencias, 7(5), 922-936.

Flórez, D., \& Ward, S. (2013). Diseño de una mini cadena productiva orgánica en San Andrés Islas a través de un itinerario de ruta como herramienta de gestión e integración. Ciencia y Tecnología Agropecuaria, 14(2), 129-147.

García de la Figal, A. (2018). Teoría y Metodología de la Investigación Científica. Alfaomega.

Guallpa, M., Lara, N., Espinoza, M., Guilcapi, E., y Fosado, O. (2019a). Valoración cualitativa de una plantación de Eucalyptus globulus Labill en el sector de Licto, Riobamba, Ecuador. Polo del Conocimiento, 4(4), 126-152.

Guallpa, M., Guilcapi, E., y Espinoza, A. (2019b). Flora apícola de la zona estepa espinosa Montano Bajo, en la Estación Experimental Tunshi, Riobamba, Ecuador. Dominio de las Ciencias, 5(2), 71-93.

Guallpa, M., Espinoza, A., Caranqui, J., y Guilcapi, E. (2020). Potencial melífero de dos apiarios ubicados en los cantones Cevallos y Riobamba, Zona 3 interandina, Ecuador. Conciencia Digital, 03(4.1.), 46-61.

May, T., y Rodríguez, S. (2012). Plantas de interés apícola en el paisaje: Observaciones de campo y la percepción de apicultores en República Dominicana. Revista Geográfica de América Central, 48 (1), 133-162.

Ministerio de Agricultura, ganadería, acuacultura y pesca [MAGAP]. (2015). Ecuador tiene potencial para la apicultura. https://www.agricultura.gob.ec/ecuador-tienepotencial-para-la-apicultura/

Montenegro, G. (2013). Innovación y valor agregado en los productos apícolas. Diferenciación y nuevos usos industriales. Oficina de Estudios y Políticas Agrarias (ODEPA).

Nates, G. (2016). Iniciativa Colombia de polinizadores: abejas ICPA. Instituto Humboldt.

Pinilla, M., y Nates, G. (2015). Diversidad de visitantes y aproximación al uso de nidos trampa para Xylacopa (Hymenoptera: Apidae) en una zona productora de pasiflora en Colombia. Actualidades Biológicas, 37(103), 143-153. 
Quezada, N. (2017). Estadística con SPSS 24. Macro.

Sánchez, C. (2013). Crianza y producción de abejas-apicultura. Ripalme.

Sierra, R., Cerón, C., Palacios, W., y Valencia, R. (1999). Propuesta preliminar de un sistema de clasificación para el Ecuador Continental. Proyecto INEFAN/GEFBIRF y EcoCiencia.

Silva, L; y Restrepo, S. (2012). Flora Apícola. Determinación de la oferta floral apícola como mecanismo para optimizar la producción, diferenciar producto de la colmena y mejorar la competitividad. Instituto de investigación de recursos biológicos Alexander Von Humboldt.

Trópicos.Org. Missouri Botanical Garden. (2020). www.tropicos.org

Zavala, J. (2013). Estudio de mieles diferenciadas de México y Guatemala y su potencial para la conservación de la biodiversidad. Tesis de Doctorado en Ciencias en Ecología y Desarrollo Sustentable, El Colegio de la Frontera Sur, México. 
El artículo que se publica es de exclusiva responsabilidad de los autores y no necesariamente reflejan el pensamiento de la Revista Alfa Publicaciones.

\section{LCiencia}

El artículo queda en propiedad de la revista y, por tanto, su publicación parcial y/o total en otro medio tiene que ser autorizado por el director de la Revista Alfa Publicaciones.
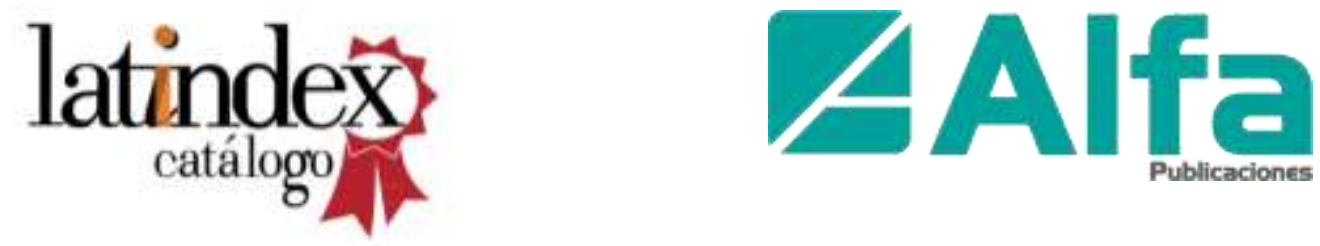

doi crossrot latindex URKUND Google RəAD DLatinREV ESJI $=$ REDIB $\underbrace{}_{\text {wizdom.ai }} \bigcirc_{\text {OpenAlRE }}^{+}$

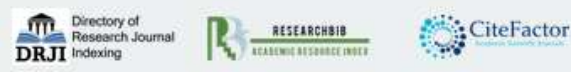

